\title{
Competencias médicas requeridas para el buen desempeño en Centros de Salud Familiares en Chile
}

\author{
Joaquín Montero ${ }^{1}, M_{\text {Paulina Rojas }}^{1}$, Josette Castel ${ }^{1}$, \\ Mónica Muñoz ${ }^{2 a}$, Astrid Brunner ${ }^{3 a}$, Yuvitza Sáez ${ }^{4 b}$, \\ Judith Scharager $4 \mathrm{~b}$.
}

\section{A survey about the competences required by primary health physicians in Chile}

Background: Primary health care is considered the cornerstone of health care in Chile. Its efficiency is strongly influenced by the quality and competences of physicians that are responsible for health care at this level. Aim: To define the features and competences that should have primary care physicians. Material and methods: A group of experts that could be physicians or other professionals working in primary health care were invited to answer electronically a structured questionnaire containing a list of 71 competences, for a basic and an expert level of physician, using the Delphi method. Competences were classified as "desirable" or "indispensable". If there was lack of consensus in the importance given to a specific competence, the researchers defined its importance. Results: Thirty eight professionals (50\% physicians) were invited to participate and 16 answered the questionnaire. The competence profile defined for basic physicians has 13 knowledge items, 24 skills and 16 attitudes. The figures for advanced physicians are 29 knowledge items, 37 skills and 20 attitudes. Conclusions: This list of competences should be considered by medical schools to adapt undergraduate training of future physicians (Rev Méd 2009: 137: 1561-8).

(Key w ords: Delivery of health care; Primary health care; Professional competence)

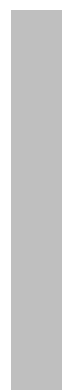

Recibido el 18 de marzo, 2009. Aceptado el 27 de octubre, 2009.

Investigación realizada en el marco del Concurso de Políticas Públicas 2007 que organizó la Pontificia Universidad Católica de Chile con el respaldo de la Cámara de Diputados, el Ministerio Secretaría General de la Presidencia, la Subsecretaría de Desarrollo Regional y Administrativo dependiente del Ministerio del Interior y la Biblioteca del Congreso Nacional.

${ }^{1}$ Departamento de Medicina Familiar, Facultad de Medicina, Pontificia Universidad Católica de Chile. ${ }^{2}$ Escuela de Enfermería, Facultad de Medicina Pontificia Universidad Católica de Chile. ${ }^{3}$ Red de Salud, P. Universidad Católica de Chile. ${ }^{4}$ Escuela de Psicología, Pontificia Universidad Católica de Chile. Santiago de Chile.

aEnfermera Universitaria

bPsicólogo

Correspondencia a: Dr. Joaquín Montero. Departamento de

Medicina Familiar, Facultad de Medicina, Pontificia Univer-

sidad Católica de Chile. Marcoleta 347 Santiago, Chile.

E mail: jmontero@med.puc.cl 
$\mathrm{L}$ a importancia de la atención primaria de salud (APS) es reconocida universalmente y ha sido corroborada recientemente en el último informe sobre la salud del mundo de la Organización Mundial de la Salud (OMS) ${ }^{1}$. Los países con APS sólida exhiben una mejor salud a costos más bajos que aquellos con una APS más débil ${ }^{2}$.

La reforma iniciada en Chile a principios de la década actual intenta poner la APS como eje, buscando construir un sistema de salud centrado en ella, en consonancia con las propuestas de la Organización Panamericana de la Salud (OPS) y $\mathrm{OMS}^{1,3}$. Para esto se requiere trabajar con equipos de salud renovados, capaces de asumir el cuidado de la salud de las personas y sus familias, en el escenario epidemiológico actual en el que se agregan los problemas emergentes como salud mental, enfermedades crónicas y envejecimiento de la población.

Distintos actores plantean la falta de profesionales idóneos como un obstáculo para enfrentar este desafío. La Academia de Medicina del Instituto Chile $^{4}$, en un reciente informe sobre la situación de la enseñanza de la medicina resalta la falta de interés de los médicos formados en Chile por trabajar en la APS y recomienda a las Facultades de Medicina que evalúen si están capacitando realmente a sus egresados para ella. Román ${ }^{5}$ recientemente constata la carencia de médicos para la APS en Chile y analiza las posibles causas de este hecho: considera por una parte la carencia de políticas de desarrollo del recurso humano (falta de plazas, carencia de carrera funcionaria e incentivos adecuados); por otra parte, anota fallas del sistema formador, por su predominio hospitalario e insuficiencias curriculares.

El objetivo de este trabajo es contribuir al desarrollo de la Atención Primaria a través de la definición de las características que deben poseer los médicos para desempeñarse adecuadamente en APS hoy en Chile, según lo propuesto por el Ministerio de Salud (MINSAL) como "Modelo de Atención Integral con Enfoque Familiar"6, el que se caracteriza por los siguientes elementos:

a) Enfoque que incorpora las dimensiones biológica, psicológica, social y familiar, incluyendo intervenciones integrales dirigidas a la familia. b) Población a cargo, se espera que exista una identificación estrecha entre el equipo de salud y las familias de las que son responsables. c) Continuidad del cuidado, a través de las etapas del ciclo vital, en salud y enfermedad y en los distintos componentes de la red de salud. d) Énfasis en prevención y promoción. e) Mirada transdisciplinaria, los diversos profesionales y técnicos, en sus roles complementarios son los encargados de asumir la responsabilidad del cuidado de las familias, para ello se deberán organizar en equipos de salud. f) Comunidad como sujeto, se espera incorporar a los individuos y las organizaciones en la toma de decisiones respecto a la atención de salud.

Se utilizó como modelo el enfoque por competencias. Este enfoque está siendo aplicado en la gestión de recursos humanos en muchos campos de la administración, la educación y también de la salud $^{7}$. Epstein y Hundert ${ }^{8}$ a partir de una amplia revisión de la literatura proponen la siguiente definición de las competencias médicas: "el uso habitual y juicioso del raciocinio clínico, conocimiento, habilidades técnicas y de comunicación, emociones y valores en la práctica diaria para el beneficio del individuo y la comunidad atendida". La OPS $^{3}$ define las competencias en forma más operativa como características de las personas (Conocimientos, Habilidades y Actitudes), que se ponen de manifiesto cuando ejecutan una tarea 0 realizan un trabajo en forma exitosa.

\section{MATERIAL Y MÉTODO}

Definir las caracteństicas de las personas que tienen éxito en su labor profesional, es una tarea propia de profesionales con experiencia en el área y de expertos. A un conjunto de ellos se les invitó a participar en la construcción de un listado de estas características a través del método Delphi ${ }^{9-12}$. Este, es un método cualitativo recomendable para estos procesos $^{13}$. A través de la iteración y retroalimentación controlada cada experto expresa y va conociendo los puntos de vista de los otros participantes y puede confirmar o modificar su opinión según la calidad de la argumentación. De esta forma, a través de rondas sucesivas el grupo logra alcanzar consenso. Al ser un proceso anónimo, asegura control sobre la influencia por reputación o liderazgo.

\section{a. Participantes}

Se invitó a participar a 38 expertos seleccionados por su responsabilidad docente o asistencial y 
experiencia en el ámbito de la APS. Se incluyó a directivos, académicos y profesionales clínicos pertenecientes a centros de atención primaria públicos y privados que trabajan en el modelo de salud familiar o vinculados a los centros universitarios de formación en salud familiar. Los participantes se agruparon en dos paneles: médicos (PM) y profesionales de salud no médicos (PNM).

b. Recolección de la información

1. Desarrollo del listado inicial: Para la construcción del instrumento inicial se seleccionó como modelo el Mapa de Competencias Médicas del Sistema Sanitario Andaluz ${ }^{14}$, por estar probado en un medio razonablemente similar al chileno. Se adaptó un listado de 71 competencias más específicas para la APS para ser presentadas a los expertos.

2. Construcción del consenso: El listado inicial de competencias con su respectiva definición se envió a los participantes y se les pidió que definieran el nivel de "necesidad" de cada competencia para la APS, considerándola "Deseable" o "Indispensable", según niveles de desarrollo del profesional. Se consideró "nivel básico" a un médico recién egresado o sin experiencia previa en APS chilena y "nivel avanzado" a un profesional con amplia experiencia en APS y que destaca en su unidad.
Inicialmente se consideró también un "nivel intermedio" que luego se suprimió dado que los expertos no lo diferenciaron significativamente del "avanzado". La circulación de los listados y la recepción de las respuestas de los expertos se realizó por vía electrónica. En caso de no obtenerse consenso, lo que fue más bien excepcional, los investigadores decidieron respecto al nivel de desarrollo en que quedaría definida la competencia.

\section{RESULTADOS}

De los expertos y profesionales invitados respondieron 8 por cada panel, con una tasa de respuesta de $42 \%$. El detalle de los invitados y los que participaron se presenta en las Tablas 1 y 2 .

El perfil se construyó considerando las competencias que los participantes calificaron como Indispensables para el desempeño en un determinado nivel de desarrollo.

Para el logro del consenso en el listado final de competencias el nivel avanzado requirió la circulación de dos rondas de opiniones, en cambio el nivel básico requirió de tres rondas.

Respecto del comportamiento de los paneles, el PM mostró una mayor convergencia inicial que el PNM respecto a conocimientos y habilidades

Tabla 1. Invitados y participantes en el panel médico

\begin{tabular}{|lcc|}
\hline Características de los médicos participantes & Invitados & Participantes \\
\hline Académicos & 5 & 2 \\
Directivos & 4 & 1 \\
Médicos clínicos & 8 & 3 \\
Residentes & 2 & 2 \\
Total & 19 & 8 \\
\hline
\end{tabular}

Tabla 2. Invitados y participantes panel profesionales no médicos

\begin{tabular}{|lcc|}
\hline Características de los profesionales participantes & Invitados & Participantes \\
\hline Académicos & 4 & 1 \\
Directivos & 10 & 5 \\
Profesionales clínicos & 5 & 2 \\
Total & 19 & 8 \\
\hline
\end{tabular}


para el nivel avanzado. Lo mismo sucedió para el nivel básico respecto a los conocimientos, pero hubo mayor divergencia respecto a las habilidades de este nivel. En cuanto a las actitudes, la tendencia fue de un consenso más fácil en ambos paneles para los dos niveles.

El perfil de competencias así construido para el nivel básico consta de 13 conocimientos, 24 habilidades y 16 actitudes. El perfil del nivel avanzado consta de 29 conocimientos, 37 habilidades y 20 actitudes.

A continuación se presenta el listado de competencias ordenado en 6 tablas según los elementos del modelo propuesto por el Ministerio de Salud ${ }^{6}$ para la APS. Hay que considerar que en cada listado las competencias de nivel avanzado son adicionales a las señaladas específicamente para el nivel básico.

\section{DISCUSIÓN}

Este trabajo nos entrega un listado de las competencias necesarias para un buen desempeño médico en APS bajo un modelo de salud familiar. A partir del enfoque por competencias 3,8,15-17, y usando el método Delphi se identificó un conjunto de competencias indispensables para asegurar este buen desempeño ${ }^{12}$.

La selección de los participantes considerando médicos y no médicos, de distintas posiciones laborales tanto del sector público como privado, nos aseguró una amplia representatividad.

Para evitar el sesgo entre pares y la tendencia al pensamiento grupal se usaron paneles separados de médicos y no médicos, confrontando la perspectiva médica con los no médicos, conside-

Tabla 3. Perfil de competencias del médico trabajando en APS con "Enfoque multidimensional integral"

\begin{tabular}{|c|c|c|}
\hline & Nivel Básico & $\begin{array}{l}\text { Nivel Avanzado } \\
\text { (adicionales a las del N ivel Básico) }\end{array}$ \\
\hline $\mathrm{C}$ & $\begin{array}{l}\text { - Diagnóstico, tratamiento y pronóstico } \\
\text { específicos de los problemas de salud } \\
\text { habituales en atención primaria } \\
\text { - Signos de alarma y complicaciones de } \\
\text { los problemas de salud habituales } \\
\text { en APS } \\
\text { - Desarrollo evolutivo de la familia } \\
\text { - Tratamiento farmacológico para APS }\end{array}$ & $\begin{array}{l}\text { - Fisiopatología de los problemas } \\
\text { habituales APS } \\
\text { - Psicopatología de los problemas más } \\
\text { frecuentes de salud mental } \\
\text { - Escala de valoración de discapacidad } \\
\text { y evaluación del riesgo } \\
\text { - Evaluación del ámbito familiar } \\
\text { - Estrategias de intervención familiar } \\
\text { - Cuidados paliativos }\end{array}$ \\
\hline & - Enfoque integral multidimensional & $\begin{array}{l}\text { - Técnicas e instrumentos psicosociales } \\
\text { en el ámbito familiar }\end{array}$ \\
\hline $\mathrm{H}$ & $\begin{array}{l}\text { - Diagnóstico clínico con las herramientas } \\
\text { de la APS } \\
\text { - Atención médica de emergencia } \\
\text { - Técnicas de soporte vital avanzado }\end{array}$ & $\begin{array}{l}\text { - Técnicas diagnósticas y terapéuticas } \\
\text { específicas y exploraciones complementarias } \\
\text { apropiadas a la atención primaria }\end{array}$ \\
\hline A & $\begin{array}{l}\text { - Visión de atención de salud como } \\
\text { un derecho } \\
\text { - Enfoque integral biopsicosocial del } \\
\text { individuo y respeto por la vida } \\
\text { - Empatía y asertividad }\end{array}$ & \\
\hline
\end{tabular}

C: Conocimientos; H: Habilidades; A: Actitudes 
Tabla 4. Perfil de competencias del médico trabajando en APS respecto a "Población a cargo"

\begin{tabular}{|c|c|c|}
\hline & Nivel Básico & $\begin{array}{c}\text { Nivel Avanzado } \\
\text { (adicionales a las del N ivel Básico) }\end{array}$ \\
\hline C & $\begin{array}{l}\text { - } \text { Situación de salud y sus determinantes } \\
\text { - } \text { Planificación y programación de la } \\
\text { - Princidad asistencial } \\
\text { - } \quad \text { Derechos ética profesional } \\
\text { yeberes de los pacientes }\end{array}$ & $\begin{array}{l}\text { - Epidemiología y situación de } \\
\text { salud de la población que atiende } \\
\text { - Medicina basada en la evidencia: } \\
\text { aplicabilidad } \\
\text { - Costo efectividad de las intervenciones } \\
\text { habituales } \\
\text { - Sistemas de evaluación sanitaria y } \\
\text { - Metodología de calidad } \\
\text { - Metodología de la investigación nivel básico }\end{array}$ \\
\hline $\mathrm{H}$ & $\begin{array}{l}\text { - } \quad \text { Relaciones interpersonales efectivas } \\
\text { Prionización de problemas y gestión } \\
\text { - Comunicación oral } \\
\text { - Comunicación escrita }\end{array}$ & $\begin{array}{l}\text { - Utilización de recursos disponibles } \\
\text { - Ética en el ejercicio profesional en APS } \\
\text { - Técnicas básicas de investigación }\end{array}$ \\
\hline A & $\begin{array}{l}\text { - Vocación social por el trabajo en APS } \\
\text { - Orientación a resultados y perseverancia }\end{array}$ & $\begin{array}{l}\text { - Orientación a satisfacer expectativas } \\
\text { de pacientes y familiares } \\
\text { - Visión de futuro }\end{array}$ \\
\hline
\end{tabular}

C: Conocimientos; H: Habilidades; A: Actitudes

Tabla 5. Perfil de competencias del médico trabajando en APS respecto a "C ontinuidad del cuidado"

\begin{tabular}{|c|c|c|}
\hline & Nivel Básico & $\begin{array}{c}\text { Nivel Avanzado } \\
\text { (adicionales a las del N ivel Básico) }\end{array}$ \\
\hline \multirow[t]{2}{*}{$\mathrm{C}$} & $\begin{array}{l}\text { - Proceso asistencial de medicina de los } \\
\text { problemas de salud habituales }\end{array}$ & $\begin{array}{l}\text { - Estructura, funcionamiento y beneficios } \\
\text { de la red de salud con la que le corresponde } \\
\text { interactuar respecto a los problemas } \\
\text { más frecuentes de sus usuarios } \\
\text { - Estructura y funcionamiento de la red social } \\
\text { y comunitaria con la que puede interactuar } \\
\text { respecto a los problemas más frecuentes } \\
\text { de sus usuarios } \\
\text { - Oferta de servicios y de la organización } \\
\text { del sistema público y la APS en Chile }\end{array}$ \\
\hline & $\begin{array}{l}\text { - } \quad \text { Toma de decisiones } \\
\text { - } \quad \text { Plan y y seguimitico y síntesis } \\
\text { problemas habituales en APS }\end{array}$ & \\
\hline $\mathrm{H}$ & $\begin{array}{l}\text { - Motivación a la adherencia al tratamiento } \\
\text { - } \quad \text { Tecnologías e informática, nivel usuario } \\
\text { - } \text { en registro utilizados en AP }\end{array}$ & \\
\hline A & - Aprendizaje y mejora continua & $\begin{array}{l}\text { - Visión continua, longitudinal e integral } \\
\text { de los procesos }\end{array}$ \\
\hline
\end{tabular}

C: Conocimientos; H: Habilidades; A: Actitudes 
Tabla 6. Perfil de competencias del médico trabajando en APS respecto a "Énfasis en prevención y promoción”

\begin{tabular}{|c|c|c|}
\hline & Nivel Básico & Nivel Avanzado \\
\hline C & $\begin{array}{l}\text { - Principios de promoción de la salud, } \\
\text { educación para la salud y } \\
\text { estilos de vida saludables } \\
\text { Prevención y detección precoz de los } \\
\text { problemas de salud habituales en APS }\end{array}$ & $\begin{array}{l}\text { - Epidemiología y situación de salud } \\
\text { de la población que atiende }\end{array}$ \\
\hline $\mathrm{H}$ & $\begin{array}{l}\text { - Uso de herramientas básicas de prevención } \\
\text { con el paciente y familia para el manejo } \\
\text { de sus riesgos y para el autocuidado } \\
\text { - Uso de herramientas para la promoción } \\
\text { en salud }\end{array}$ & $\begin{array}{l}\text { - Intervenciones de educación grupal } \\
\text { y colectivas para la prevención } \\
\text { y promoción salud } \\
\text { - Docentes para actuar con su equipo } \\
\text { y con los pacientes }\end{array}$ \\
\hline A & - $\quad$ Responsabilidad & \\
\hline
\end{tabular}

C: Conocimientos; H: Habilidades; A: Actitudes

Tabla 7. Perfil de competencias del médico trabajando en APS respecto a "M irada transdisciplinaria para asumir el cuidado de las familias, en equipos de salud"

\begin{tabular}{|c|c|c|}
\hline & Nivel Básico & $\begin{array}{l}\text { Nivel Avanzado } \\
\text { (adicionales a las del Nivel Básico) }\end{array}$ \\
\hline & - Prevención de riesgos laborales & - Principios de dirección de equipos de trabajo \\
\hline & $\begin{array}{l}\text { - } \quad \text { Compromiso y motivación } \\
\text { - Trabajo en equipo } \\
\text { - Manejo de emociones }\end{array}$ & $\begin{array}{l}\text { - Resolución de conflictos y negociación } \\
\text { - Técnicas de autocuidado y prevención } \\
\text { del estrés en el equipo }\end{array}$ \\
\hline $\mathrm{H}$ & $\begin{array}{l}\text { - } \quad \text { Flexibilidad y adaptación al cambio } \\
\text { Precauciones estándar y prevención } \\
\text { de infecciones intrahospitalarias }\end{array}$ & $\begin{array}{l}\text { Liderazgo y conducción de equipos } \\
\text { de trabajo multidisciplinarios }\end{array}$ \\
\hline & $\begin{array}{l}\text { - Sentido de pertenencia a la organización } \\
\text { e identificación con sus objetivos } \\
\text { - Respeto y valoración del trabajo } \\
\text { de los demás }\end{array}$ & - Creatividad y proactividad \\
\hline A & $\begin{array}{ll}\text { - } & \text { Flexibilidad y adaptación al cambio } \\
\text { - } & \text { Honestidad, sinceridad } \\
\text { - } & \text { Diálocontrol, autoestima, auto imagen } \\
\text { solución de conflictos }\end{array}$ & \\
\hline
\end{tabular}

C: Conocimientos; H: Habilidades; A: Actitudes

rando la importancia de estos en los equipos de APS.

El número de participantes se redujo a algo menos de la mitad de los invitados iniciales, lo cual no es sorprendente. Este hecho, si bien limita parcialmente el estudio, no lo invalida, consideran- do que un número de participantes aceptable para los expertos en el método Delphi, varía entre un mínimo de 7 y hasta un máximo de 30 personas ${ }^{18}$.

Las diferencias en el grado de dificultad para alcanzar consenso entre los distintos niveles de desarrollo y tipos de competencia permiten plantear 
Tabla 8. Perfil de competencias del médico trabajando en APS respecto a "C omunidad como sujeto que toma parte en las decisiones sobre su salud"

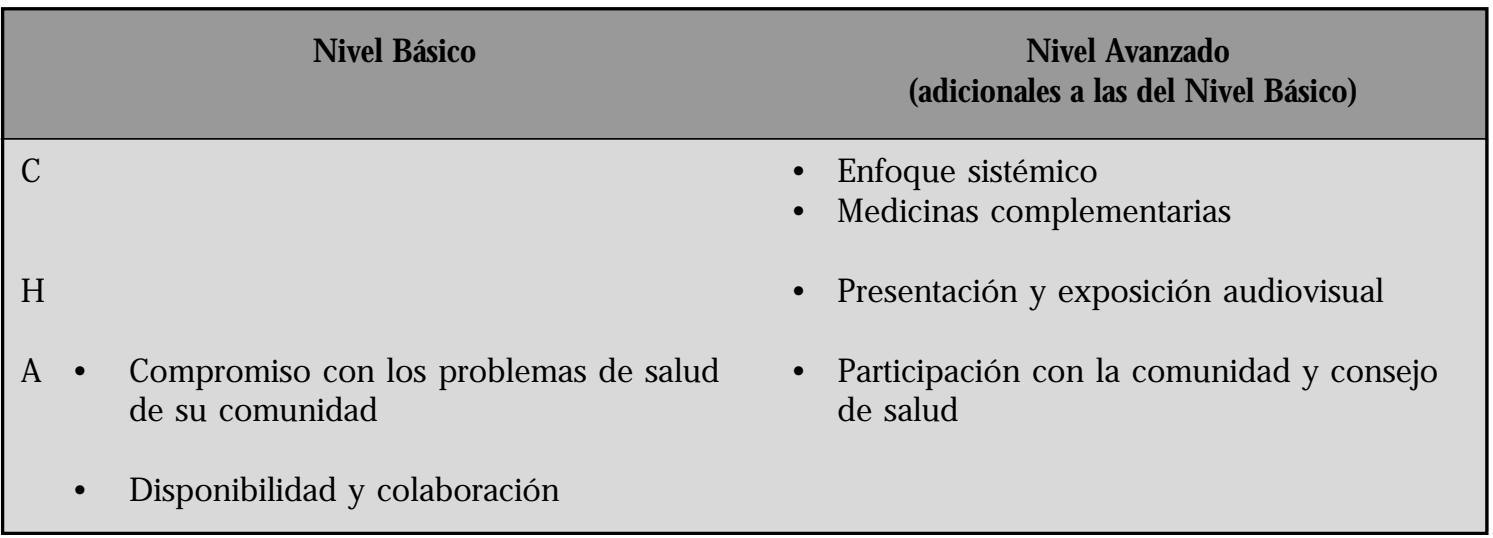

C: Conocimientos; H: Habilidades; A: Actitudes

algunas interpretaciones. El hecho que el PM haya logrado un consenso más rápido de las competencias de conocimientos que las de habilidades, podría ser reflejo de la importancia que le asignan los médicos participantes a la formación teónica. Así también la mayor dificultad para obtener el consenso en el nivel básico podría ser atribuido en parte a la realidad de la APS, que se caracteriza por una constante dificultad para cubrir sus necesidades de médicos y la coexistencia de profesionales con gran variabilidad en su formación. El nivel avanzado logra una concordancia más fácil, lo que puede ser reflejo de una meta ideal que se ha ido construyendo a través de los programas de formación de residentes y capacitación para la APS llevados a cabo por las Universidades.

La mayor facilidad para consensuar las actitudes respecto a los conocimientos y las habilidades, podría ser un reflejo del sentir vocacional de los panelistas, hallazgo frecuente de encontrar en profesionales que han optado por la APS.

Nuestra propuesta de competencias es diferente a las competencias generales definidas en el Outcome Project del Accreditation Council for Graduate Medical Education (ACGME) ${ }^{15}$, referente usado en algunas Escuelas de Medicina y que considera seis competencias genéricas para todos los programas de especialidad (Conocimiento Médico, Cuidado del Paciente, Aprendizaje y Mejoría Basada en la Práctica, Práctica Basada en Sistemas, Profesionalismo y Destrezas Interpersonales y de Comunica- ción). Estas competencias responden a un perfil general de formación de especialistas médicos; nuestra propuesta de competencias responde, más bien, a las necesidades específicas de un especialista para la APS considerando el Modelo de Atención propuesto por el Ministerio de Salud ${ }^{6}$ y las sugerencias de la OPS $^{3}$. Nuestra propuesta comparte en muchos aspectos la organización de las competencias presentada el 2005 por la WONCA europea (The World Organization of National Colleges, Academies and Academic Associations of General Practitioners/Family Physicians) ${ }^{16}$.

El listado de competencias propuesto entrega señales claras a las Facultades de Medicina para organizar una docencia de pregrado más adecuada y atractiva para la APS, concordante con las recomendaciones del informe de la Academia de Medicina $^{4}$ y recientes recomendaciones de la OPS $^{19}$.

Este estudio es un primer paso importante, que debería ser ampliado por otros estudios, para la gestión del recurso humano médico en APS, ya sea orientando la selección o mejorando la evaluación y la capacitación. Tanto el listado de competencias, como el método utilizado pueden ser una referencia para ayudar a definir las competencias de otros profesionales en el ámbito de la APS.

Este listado está pensado para ser modificado, sea corrigiendo o aportando nuevas características que permitan asegurar éxito en el desempeño médico, para así mantenerse vigente respondien- 
do a nuevas necesidades producto de los cambios del entorno y las distintas realidades locales.

El perfil de competencias del nivel avanzado refleja lo que se espera de un especialista en APS propiamente tal, el cual, trabajando con su equipo pueda ser un motor para la transformación del sector. Es decir un médico cálido, cercano a la persona y la familia, resolutivo, buen gestor de sus recursos, capaz de manejarse en la incertidumbre, liderando un equipo en forma efectiva y eficiente,

\section{REFERENCIAS}

1. Organización Mundial de la Salud. Informe sobre la salud en el mundo 2008: La atención primaria de salud, más necesaria que nunca. Ginebra, Suiza: OMS; 2008.

2. Starfield B, Shi L, MacinKo J. Contribution of primary care to health systems and health. Milbank Q 2005; 83: 457-502.

3. Organización Panamericana de la SAlud. La renovación de la atención primaria de salud de las Américas: documento de posición de la Organización Panamericana de la Salud/Organización Mundial de la Salud (OPS/OMS). Washington, USA: OPS/OMS; 2007.

4. Armas R, Goic A, Norero C, Rodríguez J, Rosselot E, Segovia S, Valdivieso V et al, 19. Informe sobre la situación actual de la educación médica en Chile. Santiago, Chile: Academia de Medicina del Instituto de Chile; 2008.

5. Román O, Pineda S, Señoret M. Perfil y número de médicos generales que requiere el país. Rev Méd Chile 2007; 135: 1209-15.

6. Ministerio de Salud. Subsecretaría de Redes Asistenciales, División de Gestión de la Red Asistencial. Modelo de Atención Integral en Salud. Santiago, Chile: MINSAL; 2005.

7. Arancibia V, Díaz R. El Enfoque de las Competencias Laborales: Historia, Definiciones y Generación de un Modelo de Competencias para las Organizaciones y las personas. Psykhe 2002; 11: 207-14.

8. EPSTEIn RM, HunderT EM. Defining and assessing professional competence. JAMA 2002; 287: 226-35.

9. BRAdiey L, Stewart K. A Delphi study of Internet banking. Marketing Intelligence \& Planning 2003; 21: 272-81.

10. Mulen P. Delphi: myths and reality. J Health Organ Manag 2003; 17: 37-52.

11. Bolger F, Rowe G, Wright G. Delphi: a reevaluation of research and theory. Technological Forecasting and Social Change 1991; 39: 235-51.

12. Rowe G, Wright G. The Delphi technique as a tolerante a la frustración, respondiendo a las necesidades múltiples que presentan las personas con una mirada integradora, utilizando ágilmente la red sanitaria, interactuando con la comunidad con las herramientas y las técnicas de las ciencias sociales y la salud pública para la prevención y promoción de la salud, preocupado no sólo de la consulta actual sino también del largo plazo, participando en la educación del equipo, las familias y la comunidad.

forecasting tool: issues and analysis. International Journal of Forecasting 1999; 15: 353-75.

13. Thompson C, Repko K, Staggers N. A Delphi study to validate competencies required of Air Force Medical Surgical nurses in mobilized environments. Mil Med 2003; 168: 618-25.

14. Junta de Andalucía; Consejería de salud, IAVANTE [homepage en Internet]. España: Fundación IAVANTE [consultado el 2 de mayo 2007]. Guía para la elaboración de mapas de competencias para los profesionales de salud; [aprox. 3 pantallas] Disponible en: http://www.iavantefundacion.com/portal3d/ $\mathrm{html} /$ queofrecemos/mapa.asp

15. Accreditation Council for Graduate Medical EducaTION. [homepage en Internet]. Chicago: ACGME; c2000-2009 [consultado el 8 de Diciembre de 2008]. Outcome Project; [aprox. 2 pantallas] Disponible en: http://www.acgme.org/acWebsite/home/home.asp.

16. WONCA Europa; Academia Europea de Profesores de medicina general. La definición europea de Medicina General / Medicina de Familia [monografía en Internet]. Barcelona: Semfyc ediciones; 2007 [Consultado el 8 de diciembre 2008]. Disponible en: http://www.semfyc.es/es/e-commerce/tienda/catalogo/detalle/LA_DEFINICION_EUROPEA_DE_MEDICINA_GENERĀL MEDICINA_DE FAMILIA/.

17. FRANK JR. The CanMEDS project: the Royal College of Physicians and Surgeons of Canada moves medical education into the 21st century. En: Dinsdale HB, Hurteau G, editores. The evolution of specialty medicine. Ottawa: Royal College of Physicians and Surgeons of Canada; 2004; 187-211.

18. Dalkey N, Brown B, Cochran S. The Delphi Method, III: Use of self rating to improve group estimates. Technological Forecasting and social Change 1970; 1: 283-91.

19. Organización Panamericana de la Salud. La formación en medicina orientada hacia la Atención Primaria de Salud. Washington DC: OPS; 2008. 